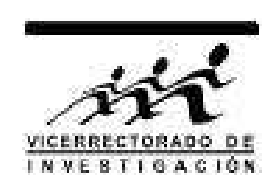

\title{
El método de Monte Carlo y la solución de la ecuación de transferencia radiativa
}

\author{
Mariella Berrocal Tito, Carlos E. Velasquez Cabrera y Raul Carita Montero \\ Facultad de Ciencias Físicas, Universidad Nacional Mayor de San Marcos, A.P. 14-0149, Lima 14, Perú
}

Recibido 24 mayo 2010 - Aceptado 20 diciembre 2010

\begin{abstract}
El paso de la radiación que atraviesa un medio donde ocurren los fenómenos de absorción, dispersión y emisión, es modelado por la ecuación de transferencia radiativa (ETR). Para medios puramente absorbentes, el fenómeno de dispersión es despreciado reduciendo la ETR a una ecuación diferencial lineal, con solución analítica. Para medios dispersivos, la ETR es una ecuación diferencial no lineal, sin solución analítica y su solución es aproximada por métodos numéricos determinísticos. Monte Carlo es un método estocástico, basado en probabilidades y utiliza un generador de números aleatorios para simular la ocurrencia de un evento, en este caso, el paso de la radiación por un medio. En este trabajo se utilizó el método de diferencias finitas junto con el de ordenadas discretas para resolver la ETR en una dimensión, los resultados fueron comparados con los obtenidos por el método de Monte Carlo. Algunos ejemplos de prueba son presentados, así también los programas utilizados para la solución de ambos métodos en el SciLab.

Palabras claves: Ecuación de transferencia radiativa, método de diferencias finitas, método de ordenadas discretas, método de Monte Carlo, SciLab.
\end{abstract}

\section{The Monte Carlo method and the solution of the radiative transfer equation}

The radiation pathway through a medium where occur the absorption, scattering and emission phenoma is described by the radiative transfer equation (ETR). For purely absorbing media, the dispersion phenomenon is neglected by reducing the ETR to a linear differential equation with analytical solution. For dispersive media, the ETR is a nonlinear differential equation, with non analytical solution and the solution must be approximated by deterministic numerical methods. Monte Carlo is a stochastic method based in probabilities and use random numbers to simulate the occurrence of an event, in this case, the pathways radiation in a participating media. In this study, we have used finite difference and discrete ordinates methods to solve the ETR in one dimension, the results were compared with the Monte Carlo results. Test case results are presented, also the source code used for the solution of both methods in the Scilab.

Keywords: Radiative transfer equation, finite difference method, discrete ordinates method, Monte Carlo method, Scilab.

En el presente trabajo se pretende contribuir a la enseñanza de la física aplicada en un tópico de permanente interés como lo es el transporte de radiación electromagnética a través de un medio contínuo. Esta fenomenología es estudiada desde diferentes enfoques como por ejemplo en física médica[1], física estelar, ingeniería mecánica[2], transferencia de calor[2], astrofísica, teledetección[3], física atmosférica[4], sensoramiento remoto. Los fenómenos de absorción y scattering producidos en un medio son denominados propiedades radiativas de un medio y proporcionan información importante sobre la composición y dinámica del mismo. El coeficiente de absorción puede discriminar diferentes componentes cromóforos, partículas que absorben radiación electromagnética de una determinada longitud de onda y que son responsables de las sustancias de coloración de un medio. El coeficiente de scattering puede ser usado para caracterizar la forma, el tamaño y la concentración de los diferentes componentes de scattering presentes en el medio[5]. Por motivos estrictamente pedagógicos,

*mabet99@yahoo.com 
mostramos el desarrollo unidimensional de la ecuación de transferencia radiativa y lo resolvemos mediante el uso de los métodos de diferencias finitas y de ordenadas discretas cuyos resultados son comparados con los obtenidos mediante el método de Monte Carlo.

\section{La ecuación de transferencia radiativa}

La ecuación de transferencia radiativa (ETR) es obtenida de la ecuación de transporte de Boltzmann[5,6] y describe el paso de las partículas neutras en un material. La ETR se describe como

$$
\begin{gathered}
\frac{1}{\boldsymbol{v}} \frac{\partial}{\partial t} I+\hat{\Omega} \cdot \nabla I+\sigma_{t} I=Q \\
Q=\int_{\Omega} \int_{0}^{\infty} \sigma_{s} I d E^{\prime} d \Omega+S \\
I=I(\boldsymbol{r}, E, \hat{\Omega}, t) \\
\sigma_{t}=\sigma_{t}(\boldsymbol{r}, E, \hat{\Omega}, t) \quad \sigma_{t}=\sigma_{s}\left(\boldsymbol{r}, E^{\prime} \rightarrow E, \hat{\Omega}, t\right) \\
S=S(\boldsymbol{r}, E, \hat{\Omega}, t)
\end{gathered}
$$

donde $I$ es la intensidad de la radiación, $\sigma_{t}$ es el coeficiente de extinción total dado por $\sigma_{t}=\sigma_{s}+\sigma_{a}$, siendo $\sigma_{a}$ el coeficiente de absorción y $\sigma_{s}$ el coeficiente de scattering, $S$ es una o más fuentes de partículas dentro del medio, $\boldsymbol{r}=(x, y, z)$ es la posición, $E$ es la energía, $\hat{\Omega}$ es el ángulo sólido de radio unitario en la dirección de propagación de la partícula y $t$ es el tiempo.

En una geometría cartesiana para un medio unidimensional en régimen permanente, considerando simetría azimutal, sin dependencia espectral, para un medio isotrópico, sin fuentes tiene la forma

$$
\begin{gathered}
\mu_{l} \frac{d I_{l}}{d x_{l}}+\sigma_{l} I_{l}=\frac{1}{4 \pi} \sum_{m=1}^{L_{0}} \sigma_{s} w_{m} I_{m} \\
l=1,2,3, \ldots, L_{0} ; \quad 0 \leq x \leq L \quad \text { y } \quad-1 \leq \mu_{l} \leq 1
\end{gathered}
$$

donde $x$ es la variable espacial, $\mu_{l}$ es el coseno de la dirección $l$ con el eje $x, w_{l}$ es el peso de la cuadratura asociada a la dirección $l, L_{0}$ es el número total de direcciones en que se divide el dominio angular de la radiación, $l$ indica una dirección.

La Fig.1, representa el problema físico a resolver en una placa larga de espesor $L_{x}$, mientras que, la Fig.2 muestra la posición de la fuente de intensidad $I_{b}$ y dos detectores, uno de reflexión, $D^{r}$ y el otro de transmisión $D^{t}$.

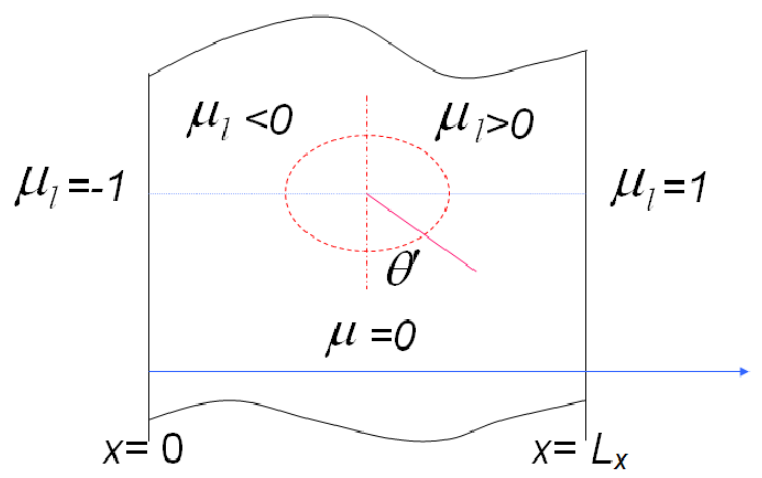

Figura 1: Medio en una dimensión.

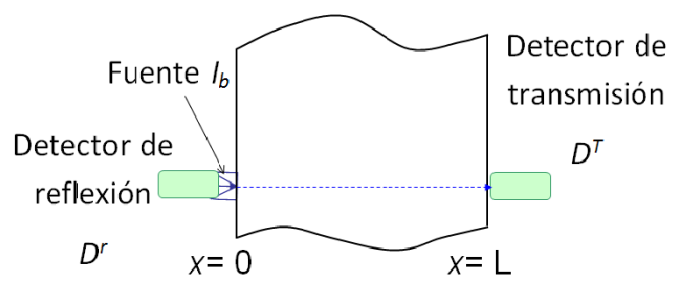

Figura 2: Posición de la fuente y los detectores.

\section{Solución analítica de la ETR}

La Ec.(2) es una ecuación diferencial no lineal que no tiene solución analítica. Pero, en el caso de que el medio fuera muy absorbente $\sigma_{s}$ puede ser despreciado, entonces se obtiene[7-9]

$$
\frac{d I}{d x}+\sigma_{a} I(x)=0
$$

La solución analítica para la Ec.(3) está dado por

$$
I(x)=I(0) \exp \left[-\sigma_{a} x\right]
$$

donde $I(0)$ es la radiacion incidente.

\section{El método de Monte Carlo}

Bajo el nombre de "método de Monte Carlo" se agrupan un conjunto de procedimientos que analizan distribuciones de variables aleatorias tratando de simular una experiencia real. La importancia actual del método Monte Carlo se basa en que este método da solución a una gran variedad de problemas físico-matemáticos permitiendo un análisis, a través de simulaciones con muestreo estadístico en una computadora, de los experimentos reales antes de realizarlos[10]. Además, las aplicaciones de este método inicialmente han surgido en 
las matemáticas como método de solución para ecuaciones diferenciales parciales, integrales multidimensionales, matrices, redondeo aleatorios y las conocidas ecuaciones de Schrödinger y Laplace. Sus aplicaciones actuales se extienden a diversos campos científicos y técnicos tan variados como la física estadística, biología molecular[11], genética [11], redes de información, telecomunicaciones y finanzas[10]. La aplicación en usos específicos[12] se cuenta a los cálculos de la magnitud de emisiones de rayos cósmicos, tamaño crítico de los reactores nucleares, difusión y movimiento browniano, difusión de líquidos a través de sólidos, propiedades de retículos poliméricos, características de los recipientes necesarios para el transporte de neutrones, y dos aplicaciones de reciente interés tienen relación con las técnicas radiográficas y con la obtención de materiales poliméricos para aplicaciones biológicas.

La principal desventaja del método de Monte Carlo es la lentitud con la que converge. La rapidez de convergencia depende de la relación $\sigma / \sqrt{M}$, donde $\sigma$ es la varianza y $M$ el número de trayectorias. Para acelerar la convergencia se disminuye el valor de $\sigma$ o se incrementa el número de trayectorias. Al incrementar el número de trayectorias se incrementa el tiempo de cálculo computacional y el uso de generadores de números aleatorios más sofisticados de modo que las secuencias numéricas sean de baja discrepancia.

El método de Monte Carlo no resuelve explícitamente la ETR. La interacción de la radiación con la materia es probabilística, $\sigma_{a}$ está relacionada a la probabilidad del material de absorber una partícula y $\sigma_{s}$ a la probabilidad de esparcirla, scattering. El método de Monte Carlo simula explícitamente el fenómeno físico, siguiendo a las partículas individuales desde su creación en una fuente hasta el término de su historia ya sea al ser absorbido o esparcido por el medio y recogido por el detector de salida[12].

Considerando un medio isotrópico, en cualquier punto del medio se cumple que:

1. La partícula que es absorbida, no es esparcida, por tanto se termina su historia.

2. La partícula esparcida, que no es absorbida, ella puede ser esparcida en diferentes ángulos. La probabilidad que la partícula sea esparcida hacia adelante, será la misma que sea esparcida hacia atrás.

El programa para los cálculos respectivos está en el apéndice al final del artículo.

\section{Solución de la ETR con el método de di- ferencias finitas y de las ordenadas finitas}

El dominio espacial es dividido en $i_{\max }$ intervalos regulares de longitud $\Delta x$, cada intervalo es denominado segmento espacial $E_{i}$, ver Fig.3, $i$ indica un segmento dentro del dominio espacial. La longitud total del medio, $L_{x}$, es igual a $L_{x}=\Delta x I_{\max }$.

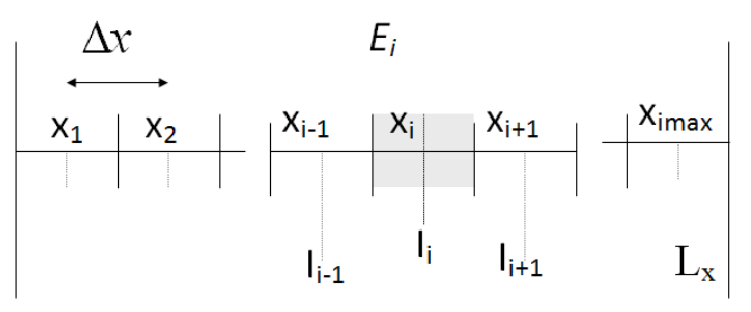

Figura 3: Discretización del domínio espacial.

Considerando los flujos $I w_{i}$ e $I e_{i}$ en los puntos de frontera del segmento $E_{i}$ como se observa en la Fig. 4, la variación de la intensidad $\Delta I$ en $E_{i}$, puede ser calculada, así como la intensidad $I_{i}$ en el centro de cada $E_{i}$ de la forma

$$
\begin{gathered}
\Delta I=I e_{i}-I w_{i} \quad \Delta x=x_{i}-x_{i-1} \\
I_{l, i}=\frac{I e_{l, i}+I w_{l, i}}{2}
\end{gathered}
$$

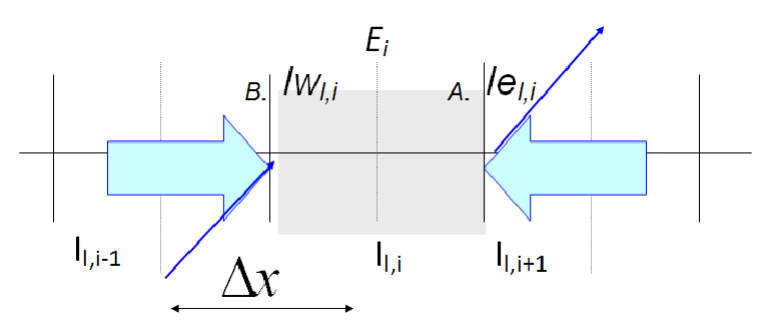

Figura 4: Aproximación por diferencias finitas.

La discretización del dominio angular, Fig. 5, es hecha en las direcciones $\Omega_{l}$ alrededor del eje $x$, ella es representada por los cosenos directores $\mu_{l}$ y sus respectivos pesos $w_{l}$ con $l=1,2, \ldots, L_{0}$. En la tabla 1, el valor $\mu_{l}$ está dentro del intervalo $-1 \leq \mu_{l} \leq 1$. Entonces el valor positivo o negativo de $\mu_{l}$ describe dos cuadrantes, Fig.6, en los cuales la radiación puede ser propagada o esparcida[13,14]. 


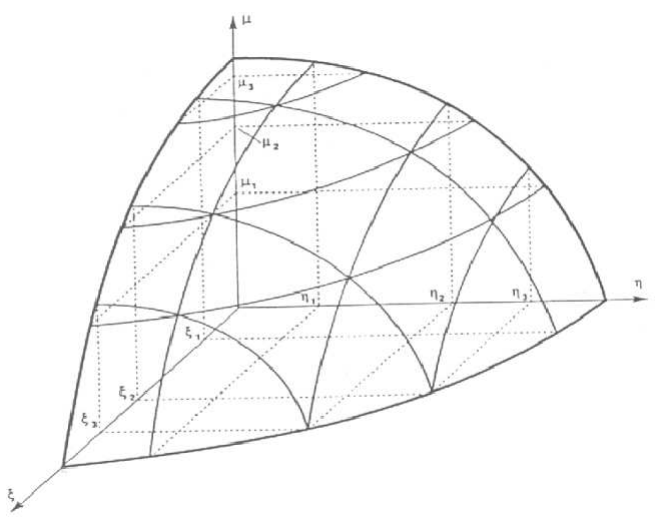

Figura 5: Discretización del dominio angular en el primer cuadrante.

Aproximando las derivadas por diferencias finitas en la Ec.(2) y ayudados de la Ec.(5), obtenemos

$$
\mu_{l} \frac{\Delta I_{1, i}}{\Delta x}+\sigma_{l, i} I_{l, i}=\mu_{l} \frac{I e_{l, i}-I w_{l, i}}{\Delta x}+\sigma_{l, i} I_{l, i}=Q_{i}
$$

Procedemos a calcular el valor de $I_{l, i}$ para cada cuadrante, comenzando con el Cuadrante I tenemos que $I_{l, i}$ va a ser desde $I w$ a $I e$, luego de la Ec.(5) tenemos

$$
I e_{l, i}=2 I_{l, i}-I w_{l, i}
$$

Reemplazando la Ec.(7) en la Ec.(6), obtenemos

$$
\begin{gathered}
I_{l, i}=\frac{2 \mu_{l} I w_{l, i}+\Delta x Q_{i}}{2 \mu_{l}+\sigma_{t, i} \Delta x} \\
l=1,2, \ldots, L_{0} \quad i=1,2, \ldots, i_{\max } .
\end{gathered}
$$

Considerando las condiciones de frontera entre los elementos de áreas adyacentes, tenemos que si

$$
i<i_{\max } \quad \text { entonces } I w_{l, i+1}=I e_{l, i} .
$$

\begin{tabular}{ccc}
\hline $\begin{array}{c}\text { Punto } \\
\text { Número }\end{array}$ & $\begin{array}{c}\text { Ordenadas } \\
\mu_{l}\end{array}$ & $\begin{array}{c}\text { Pesos } \\
w_{l}\end{array}$ \\
\hline 1 & 0.1372719 & 2.0122 \\
2 & 0.5046889 & 2.1071 \\
3 & 0.7004129 & 0.5990 \\
4 & 0.8523177 & 1.1872 \\
5 & 0.9809754 & 0.3778 \\
6 & -0.1372719 & 2.0122 \\
7 & -0.5046889 & 2.1071 \\
8 & -0.7004129 & 0.5990 \\
9 & -0.8523177 & 1.1872 \\
10 & -0.9809754 & 0.3778 \\
\hline
\end{tabular}

Tabla 1: Discretización angular.

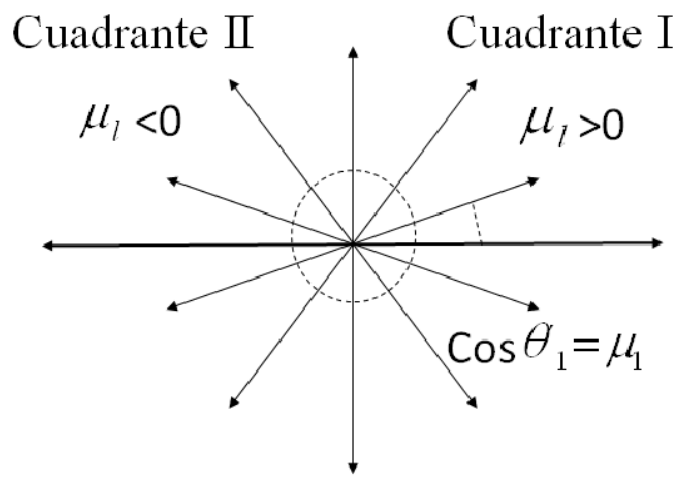

Figura 6: Cuatro cuadrantes descritos por los cosenos directores.

Cuadrante II para $\mu_{l}$, tenemos que $I_{l, i}$ va desde $I e$ a $I w$, luego obtenemos tres ecuaciones análogas al cuadrante I, dados por

$$
\begin{aligned}
I w_{l, i} & =2 I_{l, i}-I e_{l, i} \\
I & =\frac{-2 \mu_{l} I e_{l, i}+\Delta x Q_{i}}{-2 \mu_{l}+\sigma_{t, i} \Delta x} \\
I e_{l, i-1} & =I w_{l, i}
\end{aligned}
$$

Para determinar $I_{i}$ necesitamos de $Q_{l, i}$ y para calcular $Q_{l, i}$ necesitamos de $I_{i}$. Utilizando un algoritmo iterativo es posible calcular $I_{i}$ y $Q_{l, i}$. Sea $h$ un contador de iteraciones reescribimos

$$
\begin{gathered}
I_{l, i}^{h}=\frac{2\left|\mu_{l}\right| I w_{l, i}^{h}+\Delta x Q_{i}^{h-1}}{2\left|\mu_{l}\right|+\sigma_{t, i} \Delta x}, \\
\operatorname{con} \quad Q_{i}^{h-1}=\frac{1}{4 \pi} \sum_{m=1}^{L_{0}} \sigma_{s, i} w_{m} I_{m, i}^{h-1}, \\
l=1,2,3, \ldots, L_{0}
\end{gathered}
$$

El programa que corresponde a este método está disponible en un apéndice al final de artículo.

\section{Resultados}

Aplicamos los dos métodos mencionados anteriormente en tres casos que enumeramos a continuación.

Ejemplo 1 Se considera un medio homogéneo de 10 $\mathrm{cm}$ de longitud con la geometría de la Fig. 2, con una fuente de luz de $I_{0}=10,000$ fotones en un extremo y un detector de fotones en el otro. La tabla 2 muestra el número de partículas que llegan al detector, calculado a través de una solución analítica, numérica y por el método de Monte Carlo, para un medio absorbente puro con diferentes valores de $\sigma_{a}$. 


\begin{tabular}{|c|c|c|c|}
\hline & \multicolumn{3}{|c|}{ Número de fotones detectados } \\
\hline$\sigma_{a}$ & Analítica & Numérica & Monte Carlo \\
\hline \multirow{3}{*}{0.01} & \multirow{3}{*}{9048} & \multirow{3}{*}{9048} & 9044 \\
\hline & & & 9025 \\
\hline & & & 9055 \\
\hline \multirow{3}{*}{0.05} & \multirow{3}{*}{6065} & \multirow{3}{*}{6007} & 6078 \\
\hline & & & 6010 \\
\hline & & & 6024 \\
\hline \multirow{3}{*}{0.1} & \multirow{3}{*}{3679} & \multirow{3}{*}{3607} & 3653 \\
\hline & & & 3731 \\
\hline & & & 3670 \\
\hline \multirow{3}{*}{0.2} & \multirow{3}{*}{1353} & \multirow{3}{*}{1299} & 1321 \\
\hline & & & 1320 \\
\hline & & & 1330 \\
\hline \multirow{3}{*}{0.5} & \multirow{3}{*}{67} & \multirow{3}{*}{59} & 68 \\
\hline & & & 62 \\
\hline & & & 60 \\
\hline \multirow{3}{*}{0.8} & \multirow{3}{*}{3} & \multirow{3}{*}{3} & 6 \\
\hline & & & 5 \\
\hline & & & 4 \\
\hline
\end{tabular}

Tabla 2: Resultados obtenidos simulando el ejemplo 1.

La tabla 3 muestra los resultados de la solución numérica y el método de Monte Carlo, para un medio con diferentes valores de $\sigma_{s}$ y $\sigma_{a}$.

\begin{tabular}{|c|c|c|c|}
\hline \multirow[b]{2}{*}{$\sigma_{a}$} & \multicolumn{3}{|c|}{ Número de fotones en el detector } \\
\hline & $\sigma_{s}$ & numérico & Monte Carlo \\
\hline 0.5 & 0.01 & 54 & $\begin{array}{l}56 \\
75 \\
59\end{array}$ \\
\hline 0.1 & 0.05 & 2328 & $\begin{array}{l}2354 \\
2388 \\
2358\end{array}$ \\
\hline 0.2 & 0.1 & 524 & $\begin{array}{l}574 \\
604 \\
572\end{array}$ \\
\hline 0.2 & 0.2 & 209 & $\begin{array}{l}242 \\
248 \\
239\end{array}$ \\
\hline 0.1 & 0.5 & 38 & $\begin{array}{l}38 \\
42 \\
40\end{array}$ \\
\hline 0.05 & 0.8 & 4 & $\begin{array}{l}5 \\
3 \\
2\end{array}$ \\
\hline
\end{tabular}

Tabla 3: Resultados del ejemplo 1 con absorción y esparcimiento.
Ejemplo 2 Para un medio heterogéneo formado por tres capas, de modo que los extremos tienen las mismas propiedades y son diferentes a la del medio, Fig. 7. Se presenta los flujos de los fotones obtenidos al atravesar el medio mapeando las trayectorias en cada centímetro de las mismas, Fig. 8, estos resultados fueron calculados con el método de diferencias finitas, de ordenadas discretas y el de Monte Carlo. En la Fig. 9, se muestra la evolución del flujo de fotones respecto a las trayectorias recorridas calculado por el método de diferencias finitas y ordenadas discretas por cada iteración.

Ejemplo $3 \mathrm{El}$ sistema es el mismo del ejemplo 1, un medio absorbente puro, el problema se resuelve con números de historias, NH, diferentes. Los resultados mostrados en la table 4 se usaron NH iguales a $10^{4}, 10^{5}, 10^{6}$ y $10^{7}$. La Fig. 10 muestra el tiempo de cálculo computacional.

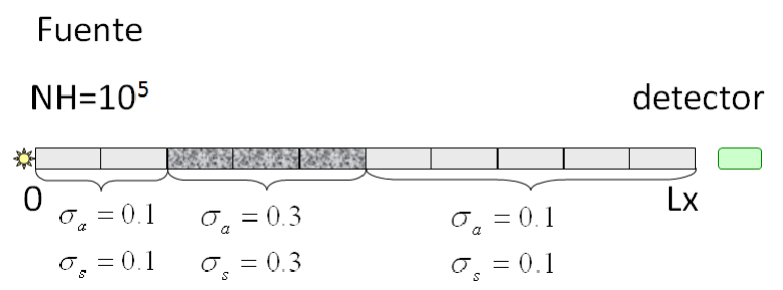

Figura 7: Medio heterogéneo considerado en el ejemplo 2.

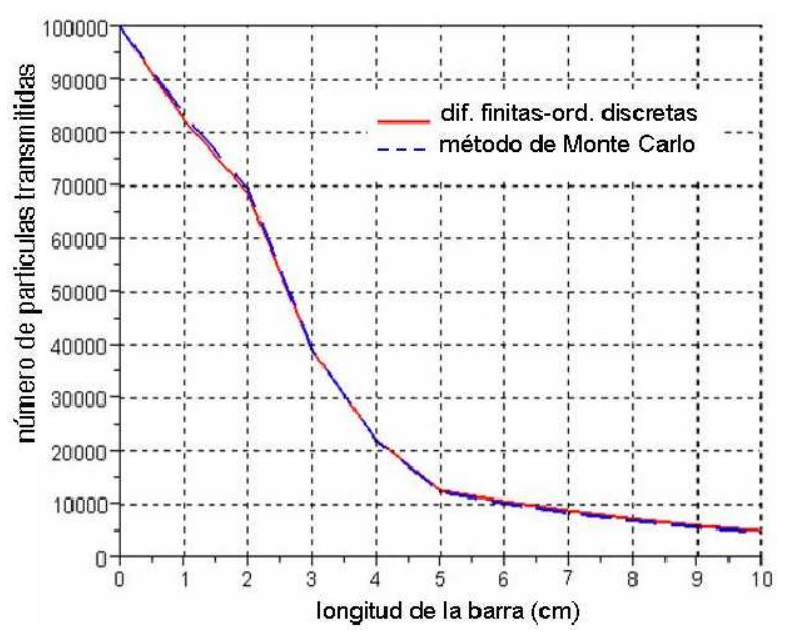

Figura 8: Medio heterogéneo analizado del ejemplo 2.

El error porcentual cuadrático medio es definido como

$$
\mathrm{EP}=100 \% \times \sqrt{\frac{1}{E} \sum_{e=1}^{E}\left(\frac{\phi_{\text {exacto }}-\phi_{e, \text { calculado }}}{\phi_{\text {exacto }}}\right)^{2}}
$$

donde $e$ indica el valor calculado en cada ejecución del programa por el método de Monte Carlo y $E$ es el número total de ejecuciones para el calculo del EP. 
En la Fig.11, se presenta el error porcentual cuadrático medio para el detector situado a la salida de un medio con $\sigma_{a}=0.8$, calculado para 4 ejecuciones del método de Monte Carlo, $\phi_{e, \text { calculado }}$ con diferentes valores de $\mathrm{NH}$, en relación a la solución analítica, valor exacto. Se considera que $E=10$ para cada valor de NH. De la Fig.11 se observa una relación entre el EP y el número de historias dada por

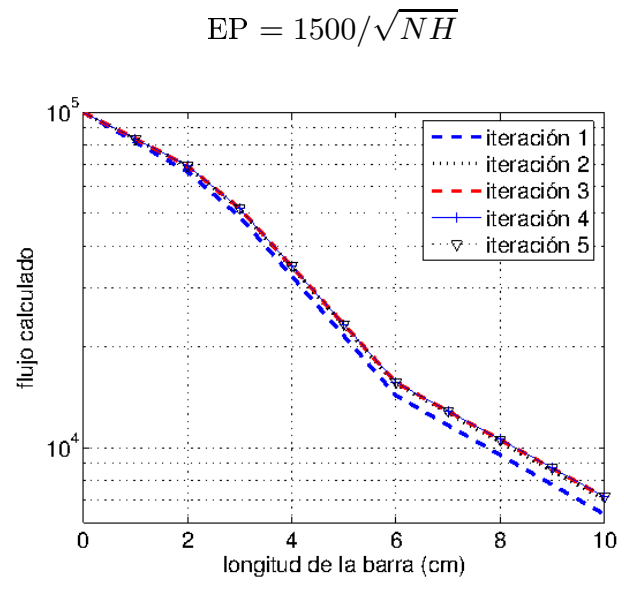

Figura 9: Aproximación de la solución en el ejemplo 2, por diferencias finitas en cada iteración.

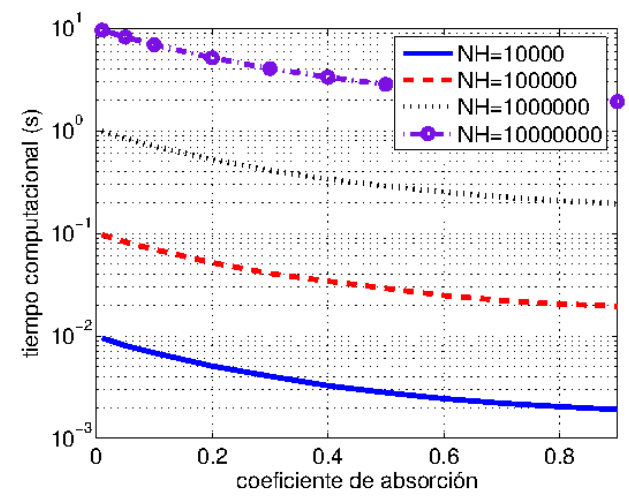

Figura 10: Tiempo de cálculo computacional para diferentes valores de $\mathrm{NH}$.

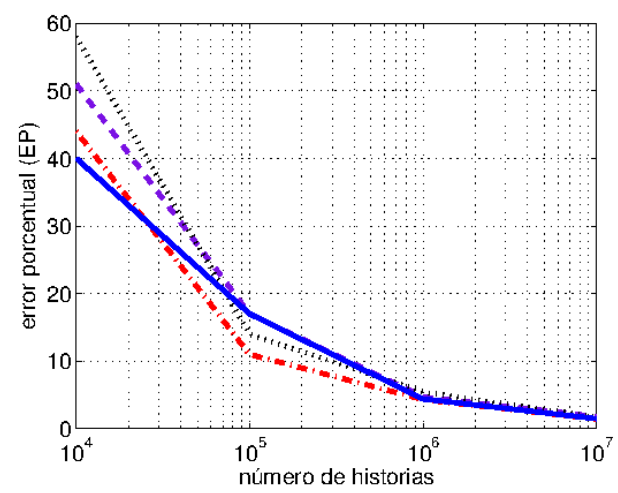

Figura 11: Error porcentual para diferentes valores de NH.

\section{Agradecimientos}

Los autores agradecen al profesor Máximo Poma Torres por las sugerencias brindadas para la realización de este trabajo.

\section{Conclusiones}

Los programas presentados en este trabajo fueron realizados en el SCILAB, un software desarrollado por el Institut national de recherche en informatique et en automatique, INRIA, de Francia[15,16].

En el método de Monte Carlo la probabilidad de que la partícula retroceda o avance por scattering en un medio isotrópico es la misma. Para determinar la probabilidad por scattering hacemos una aproximación, presentada en el programa 1, que proporciona buenos resultados.

Los resultados obtenidos por el método de Monte Carlo y el método numérico son similares. Lo cual nos induce a continuar el análisis en dos dimensiones y resolver problemas de tomografía óptica[17] en futuros trabajos.

\begin{tabular}{|c|c|c|c|c|c|c|c|c|c|c|c|c|c|}
\hline$\sigma_{a}$ & $\begin{array}{l}\text { Solución } \\
\text { analítica }\end{array}$ & \multicolumn{3}{|c|}{$\begin{array}{c}\text { Monte Carlo } \\
\mathrm{NH}=10^{4}\end{array}$} & \multicolumn{3}{|c|}{$\begin{array}{c}\text { Monte Carlo } \\
\mathrm{NH}=10^{5}\end{array}$} & \multicolumn{3}{|c|}{$\begin{array}{c}\text { Monte Carlo } \\
\mathrm{NH}=10^{6}\end{array}$} & \multicolumn{3}{|c|}{$\begin{array}{c}\text { Monte Carlo } \\
\mathrm{NH}=10^{7}\end{array}$} \\
\hline 0.01 & 9048 & 9044 & 9025 & 9055 & 9057 & 9062 & 9065 & 9048 & 9049 & 9047 & 9048 & 9049 & 9049 \\
\hline 0.05 & 6065 & 6078 & 6010 & 60 & 6076 & 6092 & 60 & 6068 & 60 & 60 & 6066 & 60 & 606 \\
\hline 0.1 & 3679 & 3653 & 3731 & 3670 & 3667 & 3676 & 3667 & 3689 & 3679 & 3682 & 3679 & 3678 & 3682 \\
\hline 0.2 & 1353 & 1321 & 1320 & 1330 & 1353 & 1343 & 1351 & 1353 & 358 & 1352 & 1354 & 1353 & 1353 \\
\hline 0.5 & 67 & 68 & 62 & 60 & 66 & 71 & 68 & 66 & 68 & 69 & 67 & 68 & 67 \\
\hline 0.8 & 3 & 6 & 5 & 4 & 3 & 3 & 2 & 3 & 3 & 4 & 3 & 3 & 3 \\
\hline
\end{tabular}

Tabla 4: Resultados calculados para el sistema del ejemplo 1. 


\section{Apéndices}

Programa usando el método de Monte Carlo.

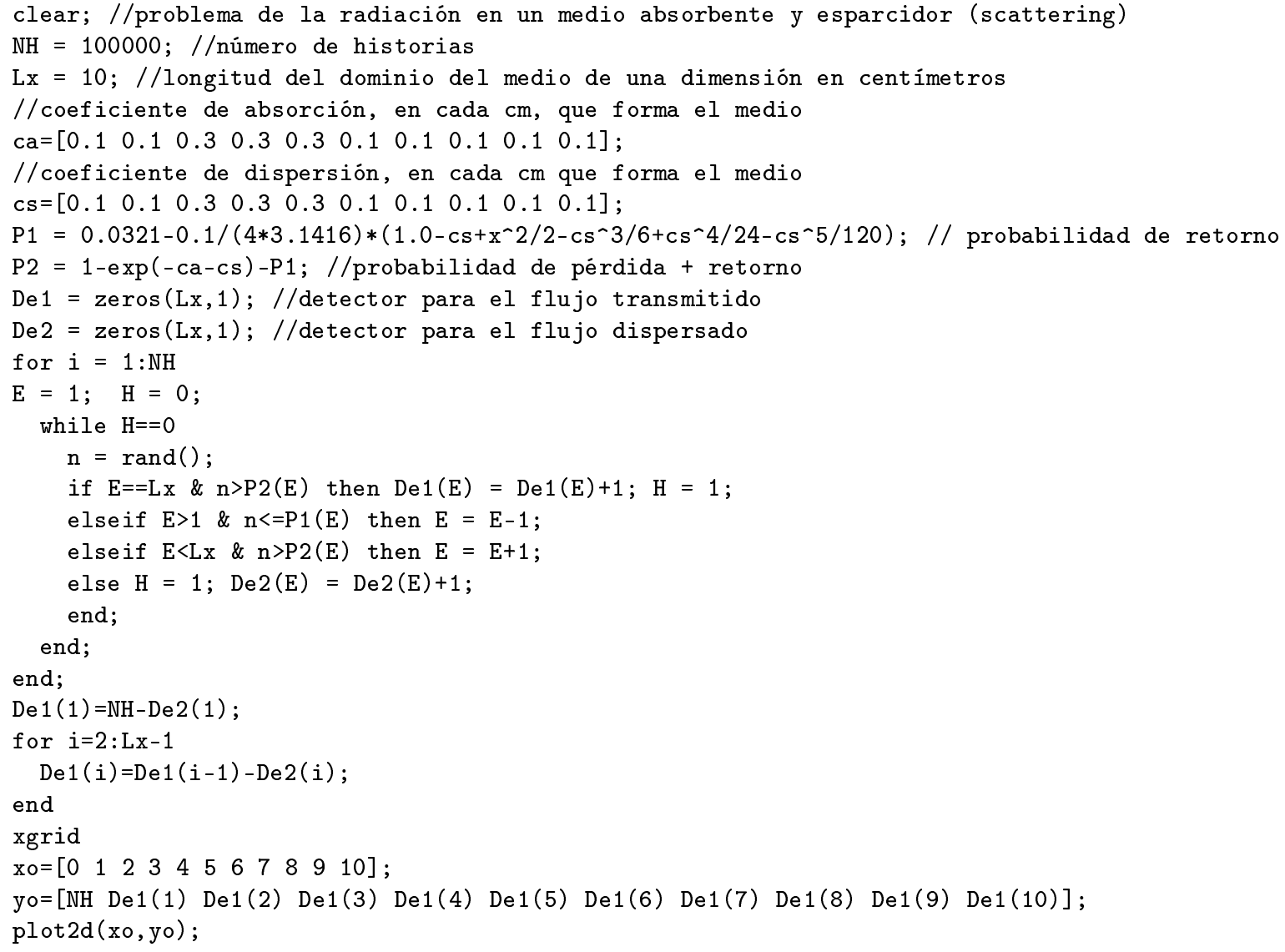

Programa usando el método de diferencias finitas y ordenadas discretas.

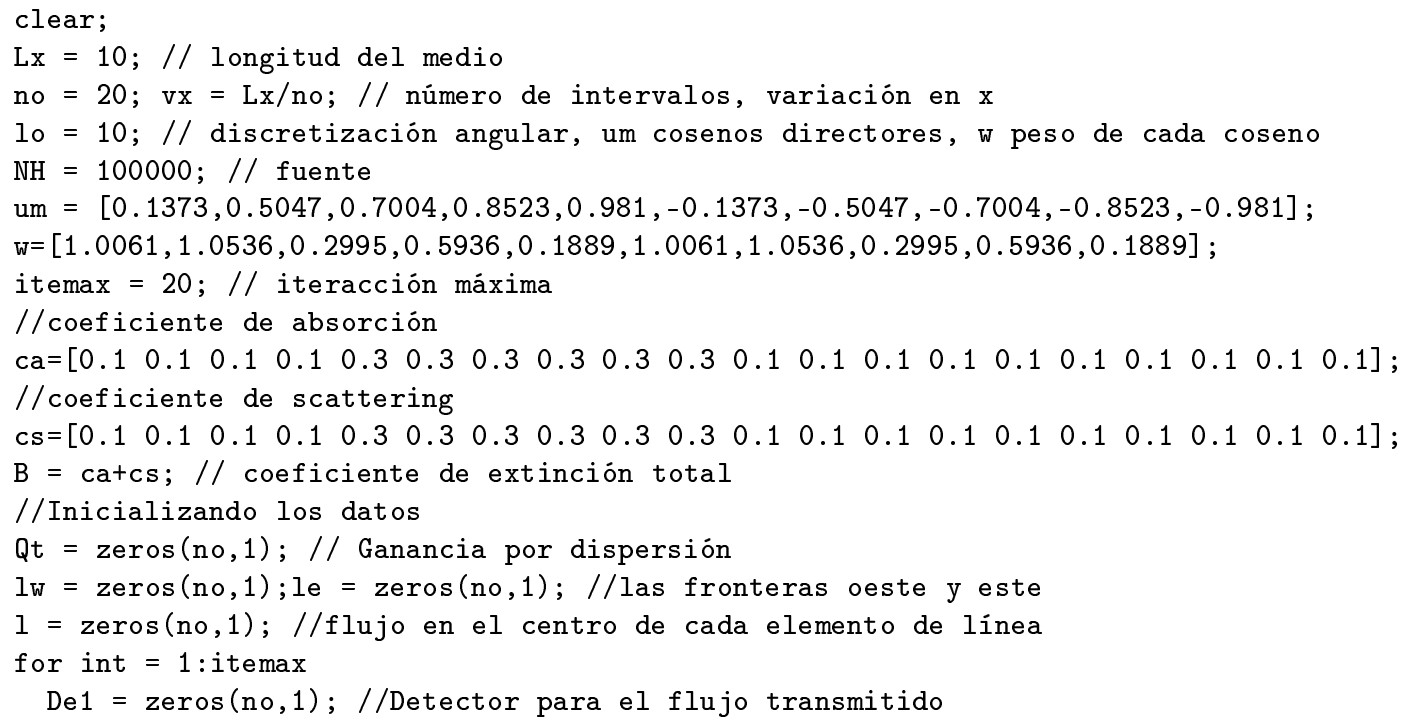




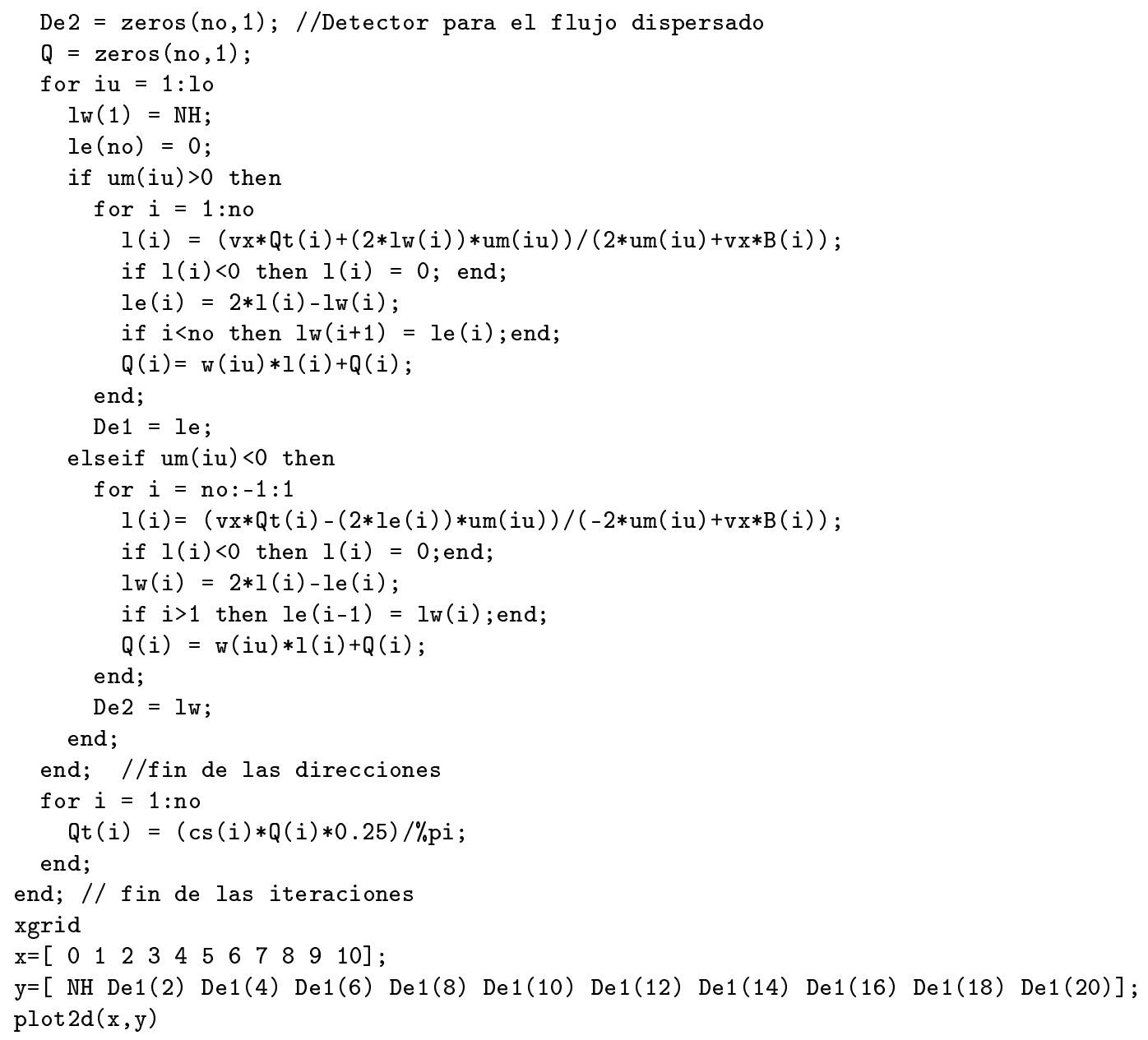

\section{Referencias}

[1] R. Elaloufi, S. Arridge, R. Pierrat, y R. Carminati; Light propagation in multilayered scattering media beyond the diffusive regime; Appl. Opt. 46, 2528 (2007).

[2] F.P. Incropera, y D.P de Witt; Fundamentos de Transferencia de Calor; Cuarta edición, John Wiley \& Sons, New York (1996).

[3] S. Gandia y J. Melia; La Teledetección en el seguimiento de los fenómenos naturales; Recursos Renovables: Agricultura, pp. 85-94, Universidad de Valencia, Valencia (1991).

[4] D. Plinio, P. Acevedo y E. Garrido; Un modelo de radiación UV desde medidas espectroradiométricas, Rev. Charlas de Física, 18, 33 (2004).
[5] J.J. Duderstadt y W.R. Martin; Trasnport theory, John Wiley \& Sons, New York (1979).

[6] F.M. Modest; Radiative heat transfer, Elsevier Science, California (1993).

[7] M.J. Berrocal, N.C. Roberty, A.J. da Silva y J. Bravo, Inverse radiative transfer problems in twodimensional participating media, Inverse Problems in Science and Engineering 12, 103 (2004).

[8] S. Chandrasekhar; Radiative transfer, Dover Publications, New York, (1960).

[9] W.A. Fiveland; Discrete ordinates solution of the radiative transfer equation for rectangular enclosure, J. Heat transfer, 106699 (1984).

[10] P. Saveedra y V.H. Ibarra; El método Monte Carlo y su aplicación a finanzas. Primer Coloquio de 
Matemáticas de la UAM-Iztapalapa. Enero 2007, docencia.izt.uam.mx/psb/coloq.pdf.

[11] J. González, M. Pérez y J. Méndez, Simulación del esparcimiento de la luz en medios biológicos mediante el método de Monte Carlo, Ingeniería Revista Académica 12, 57 (2008).

[12] X-5 Monte Carlo Team, MCNP; A general Monte Carlo N-particle transport code, Version 5, Volume I: Overview and Theory, Los Alamos National Laboratory, (2003), kevan.craufurd.org/MCNP5 .pdf.

[13] W.A. Fiveland, Discrete ordinates methods for radiative heat transfer in isotropacally and anisotro- pically scattering media, J. Heat Transfer 109, 809 (1987).

[14] K.D. Lathrop, Ray effects in discrete ordinates equation, Nucl. Sci. Eng. 32357 (1986).

[15] The Scilab Time, SCILAB 5.2, www.scilab.org.

[16] A. Alfonso y C. Valero, Fundamentos de Scilab y aplicaciones, versión 0.1, GNU Free Software Foundation (2004).

[17] S.R. Arridge, Optical tomography in medical imaging, Inverse Problems 15, R41 (1999). 